OPEN ACCESS

Edited by:

Bruno Giacomazzo,

University of Milano-Bicocca, Italy

Reviewed by:

Christian Corda,

International School of University Studies for Research and Education,

Sachiko Kuroyanagi,

Nagoya University, Japan

*Correspondence:

Patricia Schmidt

P.Schmidt@bham.ac.uk

Specialty section:

This article was submitted to

Cosmology

a section of the journal

Frontiers in Astronomy and Space

Sciences

Received: 19 February 2020

Accepted: 08 May 2020

Published: 16 June 2020

Citation:

Schmidt P (2020) Gravitational Waves

From Binary Black Hole Mergers:

Modeling and Observations.

Front. Astron. Space Sci. 7:28.

doi: 10.3389/fspas.2020.00028

\section{Gravitational Waves From Binary Black Hole Mergers: Modeling and Observations}

\author{
Patricia Schmidt * \\ School of Physics and Astronomy and Institute for Gravitational Wave Astronomy, University of Birmingham, Birmingham, \\ United Kingdom
}

Only a few years after the first detection of gravitational waves from coalescing stellar-mass black holes, the field of gravitational-wave astronomy is now firmly established after the detection of several more. These discoveries have opened up a new window onto the universe, which allows us to probe gravity and astrophysics in some of the most extreme environments in the universe. The detection and, in particular, the subsequent inference of the binaries' properties rely heavily on theoretical models of the signals. In this review, we first discuss the techniques used to model the gravitational-wave signals from coalescing black holes, before we summarize the observations made to date. We conclude with a brief outlook onto the prospects for binary black hole observations in the future.

Keywords: gravitational waves, compact binaries, black holes, modeling, observations

\section{INTRODUCTION}

The first detection of gravitational waves (GWs) from the coalescence of two stellar-mass black holes (BHs) in 2015 by the Advanced LIGO GW-detectors (Abbott et al., 2016d) marked the beginning of a new area: GW astronomy. Since then, more than ten binary black hole (BBH) mergers have been identified (Abbott et al., 2019c, 2020; Nitz et al., 2019a; Venumadhav et al., 2019a). First predicted in the 1910s by Einstein's General Theory of Relativity (GR) (Einstein, 1915, 1918), the hunt for these perturbations of spacetime itself spanned many decades and was ultimately enabled by tremendous technological and theoretical advances.

Stellar mass BBHs with a total mass between one and a few hundred solar masses, are prime GW sources for the currently operating ground-based interferometric GW detectors Advanced LIGO (Aasi et al., 2015) and Virgo (Acernese et al., 2015) as well the Japanese detector KAGRA (Akutsu et al., 2019), set to join the global GW detector network shortly. These detectors are sensitive to GWs with frequencies between $10 \mathrm{~Hz}$ to a few $\mathrm{kHz}$. In order to detect GWs in lower frequency bands, such as from the collisions of supermassive black holes in the centers of galaxies, the Earth's seismic wall needs to be overcome, which will be achieved with space-based GW missions expected to commence operation in the next decade.

The observation of GWs from inspiralling BBHs provides us with a unique means to study black holes, allowing us to perform precision tests of GR in the high-curvature, strong-field regime and shedding light on the astrophysical origin and nature of entire populations of black holes. 
Gravitational waves from BBHs carry characteristic information about the astrophysical properties of the $\mathrm{BHs}$, such as their masses and spins. These properties can be inferred via Bayesian inference (Bayes, 1764) by using highly accurate general-relativistic waveform models, which describe the last stages of the gravitationally-driven $\mathrm{BBH}$ evolution: the inspiral, the merger and the ringdown of the remnant black hole. This allows us to constrain the mass and spin distributions of stellar-mass black holes, which has implications for their possible formation history, and put constraints on the rate of such merger events in the universe.

In this review, we will first summarize the current avenues of modeling GWs from binary black holes (section 2), then discuss the current status of observations (section 3), before concluding with a very brief outlook onto the future of $\mathrm{BBH}$ GW astronomy in section 4 .

\section{MODELING BINARY BLACK HOLES}

Compact binaries, such as two black holes, on quasi-spherical orbits, lose orbital energy due to the emission of GWs, which causes their orbital separation to shrink until the two black holes plunge, merge and, in General Relativity, form a Kerr black hole. Intrinsically, a $\mathrm{BBH}$ is characterized by seven parameters: the mass ratio, $q=m_{1} / m_{2} \geq 1$, where $m_{1} \geq m_{2}$, and the two (dimensionless) spin angular momenta $\vec{\chi}_{1}$ and $\vec{\chi}_{2}$. We note that the total mass $M=m_{1}+m_{2}$ of the binary is not relevant intrinsically but determines the GW frequency in physical units and is therefore relevant for detection. Moreover, for astrophysical black holes one commonly assumes charge neutrality.

Two black holes in orbit undergo a purely gravitationallydriven evolution, which is characterized by three distinct stages: the inspiral, the merger and the ringdown of the remnant black holes. The emission of gravitational radiation causes the orbital separation to shrink. If the spin angular momenta of the two black holes are (anti-)parallel to the orbital angular momentum of the binary motion, the orbital motion is confined to a twodimensional plane whose orientation is fixed in time. In this case, the emitted GW signal is the characteristic chirp signal, a wave with monotonically increasing amplitude and frequency until the merger is reached. An example of such a waveform is shown in Figure 1. The largest amount of radiation is emitted along the direction of the orbital angular momentum (O'Shaughnessy et al., 2011; Schmidt et al., 2011), and the signal is well-described by the dominant (quadrupole) harmonic, $h_{22}$.

Any misalignment between the spins and the orbital angular momentum, however, induces relativistic precession effects, which cause the orbital plane to change its spatial orientation as the binary evolves (Apostolatos et al., 1994; Kidder, 1995). This more complex dynamics is directly reflected into the emitted GW signal, which, depending on the relative orientation w.r.t. the observer, can show strong amplitude and phase modulations (Apostolatos et al., 1994; Kidder, 1995; Schmidt et al., 2012). Further, due to the time-dependent orientation of the binary, the quadrupole approximation may no longer be sufficient to describe the radiation and higher-order harmonic modes need to be taken into account.
Due to the lack of analytic solutions of the general relativistic two-body problem, the dynamics and the corresponding GW signal must be approximated using a variety of analytic and numerical techniques. During the inspiral stage, where the orbital separation between two black holes is much larger than their extent, the BHs can be treated as point particles and their motion as well as the GW signal can be described using the post-Newtonian (PN) formalism. At small separations, however, the PN approximation is no longer valid and the Einstein field equations must be solved numerically to obtain the late-time dynamics and GW signal. In GW data analysis applications, such as matched-filter searches (Allen et al., 2012; Usman et al., 2016; Messick et al., 2017; Nitz et al., 2017; Venumadhav et al., 2019b) and Bayesian inference (Veitch et al., 2015; Zackay et al., 2018; Biwer et al., 2019), semi-analytic models with a continuous dependence on the binary parameters are most commonly used. This requires the smooth connection between $\mathrm{PN}$ and numerical relativity results. We briefly summarize the main modeling strategies below.

\subsection{Post-Newtonian Theory}

The post-Newtonian (PN) formalism is an approximation to GR valid in the slow-motion, weak-field regime (see Blanchet, 2014 and references therein for an extensive review). It is well-suited for describing the motion of compact binaries and their GW emission in the inspiral regime. In PN theory, relativistic corrections to the Newtonian solution are incorporated systematically order-by-order in the expansion parameter $\epsilon=v^{2} / c^{2}$, where $v$ is the orbital velocity and $c$ the speed of light. The PN formalism starts to break down when the orbital velocity of the black holes becomes comparable to the speed of light, i.e., $v \sim c$. At this point, one requires numerical solutions to the Einstein field equations.

\subsection{Numerical Relativity}

Solving the general relativistic two-body problem in its full generality was considered the holy grail of numerical relativity (NR) for many decades. It is only possible since the breakthroughs in 2005 (Pretorius, 2005; Campanelli et al., 2006b; Baker et al., 2007) to simulate two merging black holes and obtain the GW signal emitted in the highly dynamical, non-linear merger regime. Since the initial breakthroughs, the simulations of BBHs have become a standard tool in GW astrophysics, with ever improving accuracy and also aided by faster compute cores. To date thousands of NR simulations across the $\mathrm{BBH}$ parameter space have been performed (Mroue et al., 2013; Husa et al., 2016; Jani et al., 2016; Boyle et al., 2019; Healy et al., 2019), but the sampling is still very sparse mainly due to the high computational cost. Moreover, large mass ratio $(q \geq 20)$ and high spin magnitudes $\left(\left|\vec{\chi}_{i}\right| \geq 0.9\right)$ pose particularly challenging problems that are yet to be overcome.

Since the initial breakthrough in 2005, significant progress has been made: While the first successful simulations were those of equal-mass non-spinning BBHs spanning only the last few orbits, today simulations of aligned-spin as well as precessing quasi-circular binaries, eccentric-orbit binaries (Sperhake et al., 2008; Gold and Brügmann, 2013; Lewis et al., 2017; Hinder et al., 2018; Huerta et al., 2019; Ramos-Buades et al., 2020), and even 

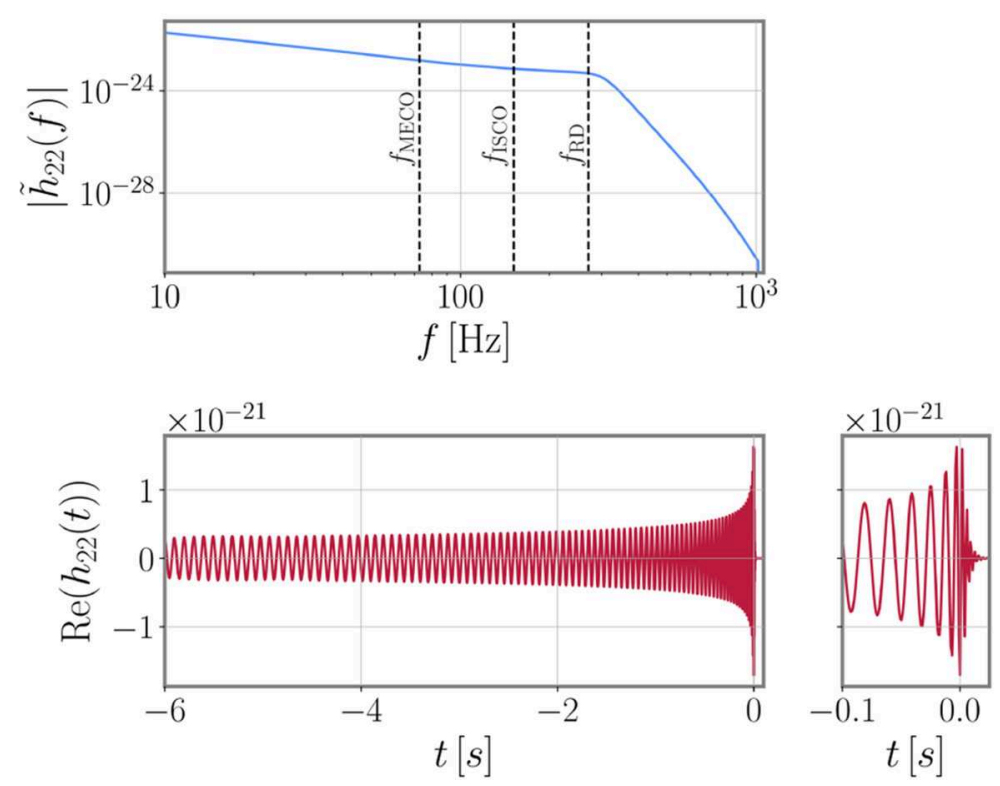

FIGURE 1 | The gravitational waveform of a non-spinning $30+30 M_{\odot}$ binary black hole at a distance of $400 \mathrm{Mpc}$ generated from the phenomenological waveform model IMRPhenomX (Pratten et al., 2020b). The top panel shows the frequency-domain GW strain starting at $10 \mathrm{~Hz}$ when viewed face-on. The vertical dashed lines indicate three characteristic frequencies: the frequency of the innermost stable circular orbit ( $\left.f_{\text {ISCO }}\right)$, the minimal energy circular orbit ( $f_{\text {MECO }}$ ) and the ringdown frequency $\left(f_{\mathrm{RD}}\right)$. The bottom panel shows the corresponding time-domain GW, where the smaller right panel shows a close-up of the last few inspiral cycles, the merger, and ringdown waveform.

evolutions that are long enough to reach into the early-inspiral regime (Szilágyi et al., 2015) are performed. Today, several codes based on different numerical techniques and formulations are capable of stably evolving $\mathrm{BBH}$ and extracting their GW signal (Campanelli et al., 2006a; Scheel et al., 2006; Sperhake, 2007; Vaishnav et al., 2007; Brügmann et al., 2008; Loffler et al., 2012; Babiuc-Hamilton et al., 2019).

Numerical relativity does not only provide the waveform through merger but also for the ringdown. The quasinormal mode spectrum emitted during this last stage of the binary evolution is analytically described by black hole perturbation theory (Teukolsky, 1973; Kokkotas and Schmidt, 1999). While the analytic prescription provides the mode frequencies and mode damping times (Berti et al., 2006), it does not provide the amplitude of the waves. Numerical relativity simulations, on the other hand, provide this crucial information, which, in combination, allows for the construction of parameter space fits (Kamaretsos et al., 2012; London et al., 2014).

The merger-ringdown waveforms obtained from NR are a key ingredient in the construction and verification of accurate waveform models used in GW data analysis. These applications, however, often require a continuous sampling across the parameter space, hence semi-analytic models or interpolants are paramount. In current analyses, waveforms from three families are most commonly employed: effective-one-body waveforms, phenomenological waveforms, and NR surrogates. While the first two paradigms model the complete inspiral-merger-ringdown (IMR) signal, NR-based surrogates are commonly restricted to a few GW cycles before the merger, the merger, and the ringdown.

\subsection{Effective-One-Body}

The effective-one-body (EOB) formalism combines information from the test particle limit as well $\mathrm{PN}$ results to obtain a complete description of the two-body dynamics as well as the IMR GW signal (Buonanno and Damour, 1999, 2000). It is based on a Hamiltonian map of the conservative dynamics of the two bodies to an effective one-body prescription, where a test particle with the reduced mass $\mu$ of the two-body system moves in an effective Kerr background spacetime characterized by the total mass $M$, the symmetric mass ratio $\eta$ and the total spin $\vec{S}$ :

$$
H=M \sqrt{1+2 \eta\left(\frac{H_{\mathrm{eff}}}{\mu}-1\right)}
$$

The EOB prescription requires that the test particle limit reduces to the motion of a particle in a Kerr spacetime and that the EOB Hamiltonian reduces to the PN Hamiltonian in the weakfield, slow-motion limit. While the Hamiltonian encapsulates the inspiral dynamics, one additionally requires a prescription for the calculation of the GWs and the radiation reaction forces as well as a smooth transition to the ringdown of a perturbed black hole. The radiative degrees of freedom are described by factorized waveforms, which include a calibration to NR simulations. We note that the EOB formalism is inherently time-domain and requires solving a system of coupled ordinary differential equations, which makes the waveform generation rather costly.

The EOB waveform family includes highly accurate quadrupolar models for non-spinning and aligned-spin binaries (Bohé et al., 2017; Nagar et al., 2018), NR-calibrated 
extensions to higher-order harmonics (Cotesta et al., 2018; Nagar et al., 2020a,b), and extensions to precessing BBH (Pan et al., 2014; Ossokine et al., 2020). Recently, extensions to include eccentric motion have been presented (Cao and Han, 2017; Hinderer and Babak, 2017).

\subsection{Phenomenological Waveforms}

The phenomenological framework takes a different approach: The focus here is exclusively on modeling the IMR GW signal itself without providing equations of motions for the $\mathrm{BH}$ dynamics (Ajith et al., 2007). The aim is to provide a fastto-evaluate, closed-form expression of the GW signal in the frequency domain by splitting the signal into an amplitude and a phase, assuming the following schematic form:

$$
\tilde{h}(f ; \vec{\lambda}, \vec{\theta})=\tilde{A}(f ; \vec{\lambda}) e^{i \tilde{\Psi}(f ; \vec{\theta})},
$$

where $\vec{\lambda}$ are (phenomenological) parameters in the amplitude and $\vec{\theta}$ in the phase respectively.

The amplitude and phase are modeled separately, where each part is subdivided into three regions: the inspiral $\left(f_{\mathrm{GW}} \leq f_{\mathrm{MECO}}\right.$, where MECO denotes the minimum-energy circular orbit), an intermediate region and the ringdown. The inspiral is modeled based on analytic PN information augmented with an artificial extension (pseudo PN terms), which are calibrated to analytic, e.g., EOB, results. The intermediate region, which governs the merger phase, is modeled as a polynomial, while the ringdown is well-described by a deformed Lorentzian. These latter two regions are calibrated to NR information. For further details see (Santamaría et al., 2010; Khan et al., 2016; Pratten et al., 2020b).

Phenomenological waveform model are constructed in the frequency domain, which makes them computationally fast to evaluate and hence particularly attractive for data analysis applications. We emphasize, however, that the validity range of any NR-calibrated waveform model depends strongly on the calibration region, which, due to the lack of NR simulations for $q \geq 10$, is limited. The most recent waveform family, PhenomX (Garcia-Quiros et al., 2020; Pratten et al., 2020b), also includes extreme mass-ratio information which allows for a smooth evaluation up to $q \simeq 1,000$ (Harms et al., 2016), although the accuracy of waveforms between $19 \leq q \leq 1,000$ cannot be assessed. The current Phenom waveform families include aligned-spin models with and without higher-order modes as well as precessing ones (Hannam et al., 2014; Khan et al., 2019; Pratten et al., 2020a). An example of the IMR waveform of an equal-mass non-spinning $\mathrm{BBH}$ in the both the frequency and the time domain is shown in Figure 1.

\subsection{Numerical Relativity Surrogates}

In recent years it has become possible to produce thousands of NR simulations for restricted parameter ranges. These relatively short waveforms can then directly be used to construct an NRbased interpolant, i.e., a surrogate model, across a (limited) volume of the binary parameter space (Field et al., 2014; Blackman et al., 2017; Varma et al., 2019a,b). Such NR-based surrogates are considered the most accurate merger models but due to their restrictions in length and parameter space coverage, their usage is currently limited.

\subsection{Gravitational Self-Force}

While moderate to high mass ratio NR simulations remain a challenge, and therefore limit the accuracy of NR-calibrated waveform models in this regime, in the extreme mass ratio limit $\left(q \geq 10^{4}\right)$ and possibly even the intermediate mass ratio regime $(q \sim 100)$, a perturbative approach, often referred to as gravitational self-force (GSF), can be used to obtain an accurate approximation (see Poisson et al., 2011; Barack and Pound, 2019 for detailed reviews). Recent progress has seen the first secondorder calculations (Pound et al., 2020), which will be crucial in order to fulfill the waveform accuracy requirements for future ground- and space-based GW observations of intermediate and extreme mass ratio binary black holes (Berry et al., 2019).

\section{OBSERVATIONS OF BINARY BLACK HOLES}

The first observation of GWs from a coalescing $\mathrm{BBH}$ by the Advanced LIGO - Virgo detector network, GW150914, in September 2015, marked the beginning of the GW discovery era. The LIGO Scientific and Virgo Collaborations have since announced a total of eleven confident detections of GWs from merging BBHs (Abbott et al., 2019c): GW150914 (Abbott et al., 2016d), GW151012 (Abbott et al., 2016b), GW151226 (Abbott et al., 2016c), GW170104 (Abbott et al., 2017a), GW170608 (Abbott et al., 2017b), GW170729, GW170809 (Abbott et al., 2019c), GW170814 (Abbott et al., 2017c), GW170818, GW170823 (Abbott et al., 2019c), and most recently GW190412 (Abbott et al., 2020). Moreover, several tens of $\mathrm{BBH}$ candidates have been identified in the currently ongoing third observing run (LIGO Scientific, Virgo Collaboration). Independent analyses of the publicly available GW strain data (LIGO Scientific Collaboration, Virgo Collaboration, 2018) have claimed the detection of additional eight binary black hole events (Nitz et al., 2019a; Venumadhav et al., 2019a; Zackay et al., 2019): Nitz et al. (2019a) reported GW170121, GW170304, GW170727, and GW151205; Venumadhav et al. (2019a) and Zackay et al. (2019) also reported GW170121, GW170304, GW170727 as well as GW151216, GW170202, GW170403 and GW170425. These observations allow us to put GR to the test in the strong-field regime (Abbott et al., 2016e, 2019d; Yunes et al., 2016), probe the astrophysics of black holes in previously unexplored mass regimes and shed light on the possible formation scenarios of black holes (Abbott et al., 2016a, 2019b).

The majority of GW events was identified via matched filtering (Sathyaprakash and Dhurandhar, 1991; Allen et al., 2012), the optimal method to extract GW signals from compact binary coalescences from noise, by comparing GR-based waveform models (see section 2) to the data. Very high-mass short-duration signals have also been identified by unmodeled searches (Klimenko et al., 2016), i.e, searches that do not rely on waveforms models but rather look for generic features. 
The black hole binaries observed through GWs to date span a wide range of total masses from just under $20 M_{\odot}$ for GW170608 (Abbott et al., 2017b), to more than $100 M_{\odot}$ for GW151205 (Nitz et al., 2019a) ${ }^{1}$ at the $90 \%$ credible level as shown in Figure 2. In particular, the heavier black holes have masses not previously seen in x-ray binaries (Corral-Santana et al., 2016), and they raise interesting questions regarding their possible formation process and history such as secondary mergers (Gerosa and Berti, 2017). So far, no black holes in either the lower mass gap between observed neutron star and BH masses (Özel et al., 2010; Farr et al., 2011), and the upper mass gap due to pair instability supernovae (Woosley, 2016; Marchant et al., 2018), have been identified. Further, most BBH observations are consistent with equal mass or marginally unequal mass binaries (Abbott et al., 2019c; Nitz et al., 2019a; Venumadhav et al., 2019a) but recently, the first BBH with an asymmetric mass ratio $(q \sim 0.28)$ has been reported (Abbott et al., 2020).

Placing precise constraints on the spins of the black holes is more difficult with the best measured spin parameter being $\chi_{\text {eff }}$, a mass-weighted linear combination of the dimensionless spin components along the orbital angular momentum direction $\hat{L}$ (Racine, 2008; Ajith et al., 2011),

$$
\chi_{\text {eff }}=\frac{\left(m_{1} \vec{\chi}_{1}+m_{2} \vec{\chi}_{2}\right) \cdot \hat{L}}{m_{1}+m_{2}} .
$$

The majority of observations is consistent with $\chi_{\text {eff }}=0$ at the 90\% credible level as shown in Figure 2, with the exceptions of: GW151226 (Abbott et al., 2016c), GW170729 (Abbott et al., 2019c), GW190412 (Abbott et al., 2020), GW151216, and GW170403 (Venumadhav et al., 2019b; Zackay et al., 2019). It should be noted, however, that the latter two events were analyzed under different spin prior assumptions. A recent reanalysis suggests that the $\chi_{\text {eff-values reported in Venumadhav }}$ et al. (2019b) and Zackay et al. (2019) for those two events may not be robust (Huang et al., 2018).

The individual black hole spin magnitudes are more difficult to constrain due to the strong dependence of the GW phase on $\chi_{\text {eff. }}$ However, a few observations find a net positive spin in the binary, implying that at least one of the two $\mathrm{BHs}$ must have a positive, non-zero spin angular momentum. Misalignment between the orbital angular momentum and the black holes spins induces relativistic precession, which is directly reflect into the GW signal (Apostolatos et al., 1994; Kidder, 1995). The observation of such precessional signatures is considered to be of key importance for distinguishing different $\mathrm{BBH}$ formation channels (Mandel and O'Shaughnessy, 2010; Rodriguez et al., 2016; Stevenson et al., 2017): BBHs formed in the field are expected to have predominantly aligned spins (Kalogera, 2000), while BBHs formed dynamically in dense environments are expected to have a more isotropic spin distribution (Rodriguez et al., 2016). In the GW observations made to date, spin misalignment remains unconstrained but it is anticipated that future

${ }^{1}$ We note that this event is considered marginal. The most significant high-mass BBH discovered to date is GW170729 (Abbott et al., 2019c).

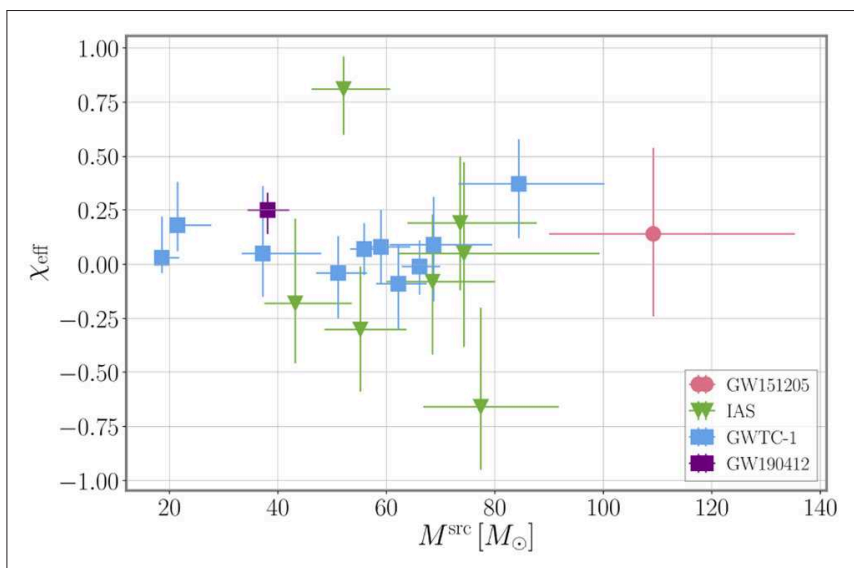

FIGURE 2 | Inferred total source-frame masses and effective inspiral spin of $\mathrm{BBH}$ observations. The error bars indicate the $90 \%$ credible interval of the $1 \mathrm{D}$ posterior probability. Blue square markers: Ten BBH events from GWTC-1 (Abbott et al., 2019c); purple square marker: GW190412 (Abbott et al., 2020); green triangular markers: events from Zackay et al. (2019) and Venumadhav et al. (2019a); red round marker: event GW151205 from Nitz et al. (2019a). Posterior samples were obtained from LIGO Scientific Collaboration, Virgo Collaboration (2018), Nitz et al. (2019b), Venumadhav et al. (2019c), and Abbott et al. (2020). The conversion from detector-frame to source-frame masses assumes a flat $\Lambda$ CDM cosmology (Ade et al., 2016).

observations will yield the first concrete measurements of precession.

Gravitational waves from merging black holes are a unique probe of GR in the strong-field high-curvature regime, and hence these observations can be used to test their consistency with the predictions from GR (Berti et al., 2015). Due to the absence of complete IMR waveforms derived in alternative theories of gravity ${ }^{2}$, we cannot directly test the validity of GR by comparison against these other theories but can perform a variety of consistency tests. One possibility is to introduce ad hoc parameterized modifications to the GR waveforms, representing either modifications in the strong-field regime or the wave propagation (Yunes and Pretorius, 2009; Mirshekari et al., 2012; Agathos et al., 2014; Berti et al., 2018b; Abbott et al., 2019d). One can then determine to which degree the inferred values of these modifications agree with the values predicted by GR. For binaries where the complete IMR signal is observed, one can further test the consistency of the inferred final mass and spin of the remnant black hole with the parameters inferred from the low- and highfrequency regimes of the signal (Hughes and Menou, 2005; Ghosh et al., 2016a,b). A detector network with non coaligned detectors allows us to investigate the presence of additional polarization states as predicted in some alternative metric theories of gravity (Eardley et al., 1973; Corda, 2009).

\footnotetext{
${ }^{2}$ Although recent progress has been made in simulating BBH mergers in alternative theories of gravity (Okounkova et al., 2019; Witek et al., 2019) and there is also ongoing effort in deriving inspiral waveforms (Sennett et al., 2016), which allow for concrete tests of alternative predictions beyond all-violations encompassing parameterized tests.
} 
Current observations show significant preference for purely tensor polarizations over purely scalar or vector polarizations respectively (Abbott et al., 2017c, 2019d). To date, all tests of GR using BBHs yield results that are consistent with GR. For current observations, the limits on deviations from GR are dominated by the statistical uncertainty due to the detector noise. Improved detector sensitivities will allow for these constraints to tighten but the impact of systematic modeling errors, such as the neglect of eccentricity (Moore and Yunes, 2020), must be carefully taken into account (Berti et al., 2015).

\section{OUTLOOK}

The observation of GWs from coalescing black holes has opened a new window onto the electromagnetically dark universe. The observations to date have already revealed the existence of black holes in previously unexplored mass regimes and have allowed us to perform the first tests of GR in a novel regime. The next generation of ground-based facilities will be significantly more sensitive than current detectors (Punturo et al., 2010; Reitze et al., 2019), allowing for even tighter measurements of black hole masses and spins, and probing the existence of stellar mass black holes throughout the history of the universe. Moreover, future observations will enable us to perform black hole spectroscopy by measuring individual quasi-normal modes (Berti et al., 2006, 2018a), probe the Kerr nature of astrophysical black holes and constrain parameterized deviations from GR in the strong-field regime to unrivaled precision (Sathyaprakash et al., 2012). Recent progress has been made calculating gravitational waveforms in alternative theories of gravity, which will allow for concrete tests of predictions beyond all-violations encompassing parameterized tests as are performed at the moment.

The detection of hundreds of $\mathrm{BBH}$ will allow for the crosscorrelations between the GW signals and galaxy catalogs will

\section{REFERENCES}

Aasi, J., Abbott, B. P., Abbott, R., Abbott, T., Abernathy, M. R., Ackley, K. et al. (2015). Advanced LIGO. Class. Quant. Grav. 32:074001.

Abbott, B. P., Abbott, R., Abbott, T. D., Abernathy, M. R., Acernese, F., Ackley, K., et al. (2016a). Astrophysical implications of the Binary Black-Hole Merger GW150914. Astrophys. J. Lett. 818:L22. doi: 10.3847/2041-8205/818/2/L22

Abbott, B. P., Abbott, R., Abbott, T. D., Abernathy, M. R., Acernese, F., Ackley, K., et al. (2016b). Binary Black Hole Mergers in the first Advanced LIGO observing run. Phys. Rev. X 6:041015. doi: 10.1103/PhysRevX.6.0 41015

Abbott, B. P., Abbott, R., Abbott, T. D., Abernathy, M. R., Acernese, F., Ackley, K., et al. (2016c). GW151226: observation of gravitational waves from a 22-solar-mass binary black hole coalescence. Phys. Rev. Lett. 116:241103. doi: 10.1103/PhysRevLett.116.241103

Abbott, B. P., Abbott, R., Abbott, T. D., Abernathy, M. R., Acernese, F., Ackley, K., et al. (2016d). Observation of gravitational waves from a binary black hole merger. Phys. Rev. Lett. 116:061102. doi: 10.1103/PhysRevLett.116.061102

Abbott, B. P., Abbott, R., Abbott, T. D., Abernathy, M. R., Acernese, F., Ackley, K., et al. (2016e). Tests of general relativity with GW150914. Phys. Rev. Lett. 116:221101. doi: 10.1103/PhysRevLett.116.221101

Abbott, B. P., Abbott, R., Abbott, T. D., Abernathy, M. R., Acernese, F., Ackley, K., et al. (2017a). GW170104: observation of a 50-solar-mass allow for an independent, precise measurement of Hubble constant $H_{0}$ (Abbott et al., 2019a), which can help shed light on the current tensions between early-time and late-time cosmological probes (Verde et al., 2019).

Furthermore, observing the mergers of $\mathrm{BBH}$ throughout the history of the universe will allow us to probe fundamental physics at a range of energy scales (Barack et al., 2019; Barausse et al., 2020). Intermediate mass black holes, in particular, have the potential to provide us with important evidences as to what the nature of dark matter may be (Eda et al., 2015; Bertone et al., 2019).

Planned space-based missions such as LISA will see a fraction of stellar mass BBHs that will later be detected by groundbased observatories while they are still in the early inspiral stage, enabling multi-band GW astronomy and providing earlywarnings to both GW and electromagnetic observatories (Sesana, 2016). This will allow us to pin-point telescopes and detect any coincident electromagnetic emission.

Gravitational-wave science has already delivered key insights into the nature and astrophysics of black holes and future observations will continue to nurture and improve our understanding of these fundamental objects in the universe.

\section{AUTHOR CONTRIBUTIONS}

PS has written this review article on her own to the best of her knowledge, she has produced all figures herself from publicly available data and codes. The references are extensive but they are by no means exhaustive.

\section{FUNDING}

PS acknowledges Dutch Research Council (NWO) Veni Grant No. 680-47-460.

binary black hole coalescence at redshift 0.2. Phys. Rev. Lett. 118:221101. doi: 10.1103/PhysRevLett.118.221101

Abbott, B. P., Abbott, R., Abbott, T. D., Abernathy, M. R., Acernese, F., Ackley, K., et al. (2017b). GW170608: observation of a 19-solar-mass binary black hole coalescence. Astrophys. J. 851:L35. doi: 10.3847/2041-8213/aa9f0c

Abbott, B. P., Abbott, R., Abbott, T. D., Abernathy, M. R., Acernese, F., Ackley, K., et al. (2017c). GW170814: A three-detector observation of gravitational waves from a binary black hole coalescence. Phys. Rev. Lett. 119:141101. doi: 10.1103/PhysRevLett.119.141101

Abbott, B. P., Abbott, R., Abbott, T. D., Abernathy, M. R., Acernese, F., Ackley, K., et al. (2019b). Binary black hole population properties inferred from the first and second observing runs of advanced LIGO and advanced Virgo. Astrophys. J. 882:L24. doi: 10.3847/2041-8213/ab3800

Abbott, B. P., Abbott, R., Abbott, T. D., Abernathy, M. R., Acernese, F., Ackley, K., et al. (2019c). GWTC-1: a gravitational-wave transient catalog of compact binary mergers observed by LIGO and Virgo during the first and second observing runs. Phys. Rev. X 9:031040. doi: 10.1103/PhysRevX.9.031040

Abbott, B. P., Abbott, R., Abbott, T. D., Abernathy, M. R., Acernese, F., Ackley, K., et al. (2019d). Tests of general relativity with the binary black hole signals from the LIGO-Virgo catalog GWTC-1. Phys. Rev. D100:104036. doi: 10.1103/PhysRevD.100.062006

Abbott, B. P., Abbott, R., Abbott, T. D., Abraham, S., Acernese, F., Ackley, K., et al. (2019a). A gravitational-wave measurement of the Hubble constant following 
the second observing run of Advanced LIGO and Virgo. arXiv [Preprint]. arXiv:1908.06060.

Abbott, R., Abbott, T. D., Abraham, S., Acernese, F., Ackley, K., Adams, C., et al. (2020). GW190412: observation of a binary-black-hole coalescence with asymmetric masses. arXiv [Preprint]. arXiv:2004.08342.

Acernese, F., Agathos, M., Agatsuma, K., Aisa, D., Allemandou, N., Allocca, A., et al. (2015). Advanced Virgo: a second-generation interferometric gravitational wave detector. Class. Quant. Grav. 32:024001. doi: 10.1088/0264-9381/32/2/024001

Ade, P. A. R., Aghanim, N., Arnaud, M., Ashdown, M., Aumont, J., Baccigalupi, C., et al. (2016). Planck 2015 results. XIII. Cosmological parameters. Astron. Astrophys. 594:A13. doi: 10.1051/0004-6361/2015 25830

Agathos, M., Del Pozzo, W., Li, T. G. F., Van Den Broeck, C., Veitch, J., and Vitale, S. (2014). TIGER: a data analysis pipeline for testing the strong-field dynamics of general relativity with gravitational wave signals from coalescing compact binaries. Phys. Rev. D 89:082001. doi: 10.1103/PhysRevD.89.082001

Ajith, P., Babak, S., Chen, Y., Hewitson, M., Krishnan, B., Whelan, J. L., et al. (2007). Phenomenological template family for blackhole coalescence waveforms. Classic. Quant. Grav. 24, S689-S700. doi: $10.1088 / 0264-9381 / 24 / 19 / \mathrm{S} 31$

Ajith, P., Hannam, M., Husa, S., Chen, Y., Brügmann, B., Dorband, N., et al. (2011). Inspiral-merger-ringdown waveforms for blackhole binaries with non-precessing spins. Phys. Rev. Lett. 106:241101. doi: 10.1103/PhysRevLett.106.241101

Akutsu, T., Ando, M., Arai, K., Arai, Y., Araki, S., Araya, A., et al. (2019). KAGRA: 2.5 generation interferometric gravitational wave detector. Nat. Astron. 3, 35-40. doi: 10.1038/s41550-018-0658-y

Allen, B., Anderson, W. G., Brady, P. R., Brown, D. A., and Creighton, J. D. E. (2012). FINDCHIRP: an algorithm for detection of gravitational waves from inspiraling compact binaries. Phys. Rev. D 85:122006. doi: 10.1103/PhysRevD.85.122006

Apostolatos, T. A., Cutler, C., Sussman, G. J., and Thorne, K. S. (1994). Spin induced orbital precession and its modulation of the gravitational wave forms from merging binaries. Phys. Rev. D 49, 6274-6297. doi: 10.1103/PhysRevD.49.6274

Babiuc-Hamilton, M., Brandt, S. R., Diener, P., Elley, M., Etienne, Z., Ficarra, G. et al. (2019). The Einstein Toolkit. To Find Out More. Available online at: http:// einsteintoolkit.org

Baker, J. G., van Meter, J. R., McWilliams, S. T., Centrella, J., and Kelly, B. J. (2007). Consistency of post-Newtonian waveforms with numerical relativity. Phys. Rev. Lett. 99:181101. doi: 10.1103/PhysRevLett.99.181101

Barack, L., Cardoso, V., Nissanke, S., Sotiriou, T. P., Askar, A., Belczynski, C., et al. (2019). Black holes, gravitational waves and fundamental physics: a roadmap. Class. Quant. Grav. 36:143001. doi: 10.1088/1361-6382/ab0587

Barack, L., and Pound, A. (2019). Self-force and radiation reaction in general relativity. Rept. Prog. Phys. 82:016904. doi: 10.1088/1361-6633/aae552

Barausse, E., Berti, E., Hertog, T., Hughes, S. A., Jetzer, P., Pani, P., et al. (2020). Prospects for fundamental physics with LISA. arXiv [Preprint]. arXiv:2001.09793.

Bayes, R. T. (1764). An essay toward solving a problem in the doctrine of chances. Philos. Trans. R. Soc. Lond. 53, 370-418. doi: 10.1098/rstl.1763.0053

Berry, C. P. L., Hughes, S. A., Sopuerta, C. F., Chua, A. J. K., Heffernan, A., HolleyBockelmann, K., et al. (2019). The unique potential of extreme mass-ratio inspirals for gravitational-wave astronomy. arXiv [Preprint]. arXiv:1903.03686.

Berti, E., Barausse, E., Cardoso, V., Gualtieri, L., Pani, P., Sperhake, U., et al. (2015). Testing general relativity with present and future astrophysical observations. Classic. Quant. Grav. 32:243001. doi: 10.1088/0264-9381/32/24/2 43001

Berti, E., Cardoso, V., and Will, C. M. (2006). On gravitational-wave spectroscopy of massive black holes with the space interferometer LISA. Phys. Rev. D 73:064030. doi: 10.1103/PhysRevD.73.064030

Berti, E., Yagi, K., Yang, H., and Yunes, N. (2018a). Extreme gravity tests with gravitational waves from compact binary coalescences: (II) ringdown. Gen. Rel. Grav. 50:49. doi: 10.1007/s10714-018-2372-6

Berti, E., Yagi, K., and Yunes, N. (2018b). Extreme gravity tests with gravitational waves from compact binary coalescences: (I) inspiral-merger. Gen. Rel. Grav. 50:46. doi: 10.1007/s10714-018-2362-8
Bertone, G., Croon, D., Amin, M. A., Boddy, K. K., Kavanagh, B. J., Mack, K. J., et al. (2019). Gravitational wave probes of dark matter: challenges and opportunities. arXiv [Preprint]. arXiv:1907.10610.

Biwer, C. M., Capano, C. D., De, S., Cabero, M., Brown, D. A., Nitz, A. H., et al. (2019). PyCBC inference: a Python-based parameter estimation toolkit for compact binary coalescence signals. Publ. Astron. Soc. Pac. 131:024503. doi: 10.1088/1538-3873/aaef0b

Blackman, J., Field, S. E., Scheel, M. A., Galley, C. R., Ott, C. D., Boyle, M., et al. (2017). Numerical relativity waveform surrogate model for generically precessing binary black hole mergers. Phys. Rev. D 96:024058. doi: 10.1103/PhysRevD.96.024058

Blanchet, L. (2014). Gravitational radiation from post-Newtonian sources and inspiralling compact binaries. Living Rev. Relativ. 17:2. doi: 10.12942/lrr-2014-2

Bohé, A., Shao, L., Taracchini, A., Buonanno, A., Babak, S., Harry, I. W., et al. (2017). An improved effective-one-body model of spinning, nonprecessing binary black holes for the era of gravitational-wave astrophysics with advanced detectors. Phys. Rev. D 95:044028. doi: 10.1103/PhysRevD.95.044028

Boyle, M., Hemberger, D., Iozzo, D. A. B., Lovelace, G., Ossokine, S., Pfeiffer, H. P., et al. (2019). The SXS Collaboration catalog of binary black hole simulations. Class. Quant. Grav. 36:195006. doi: 10.1088/1361-6382/ab34e2

Brügmann, B., González, J. A., Hannam, M., Husa, S., Sperhake, U., and Tichy, W. (2008). Calibration of moving puncture simulations. Phys. Rev. D 77:024027. doi: 10.1103/PhysRevD.77.024027

Buonanno, A., and Damour, T. (1999). Effective one-body approach to general relativistic two-body dynamics. Phys. Rev. D 59:084006. doi: 10.1103/PhysRevD.59.084006

Buonanno, A., and Damour, T. (2000). Transition from inspiral to plunge in binary black hole coalescences. Phys. Rev. D 62:064015. doi: 10.1103/PhysRevD.62.064015

Campanelli, M., Lousto, C. O., Marronetti, P., and Zlochower, Y. (2006a). Accurate evolutions of orbiting black-hole binaries without excision. Phys. Rev. Lett. 96:111101. doi: 10.1103/PhysRevLett.96.111101

Campanelli, M., Lousto, C. O., and Zlochower, Y. (2006b). Spinningblack-hole binaries: the orbital hang up. Phys. Rev. D 74:041501. doi: 10.1103/PhysRevD.74.041501

Cao, Z., and Han, W.-B. (2017). Waveform model for an eccentric binary black hole based on the effective-one-body-numerical-relativity formalism. Phys. Rev. D 96:044028. doi: 10.1103/PhysRevD.96.044028

Corda, C. (2009). Interferometric detection of gravitational waves: the definitive test for General Relativity. Int. J. Mod. Phys. D 18, 2275-2282. doi: $10.1142 / S 0218271809015904$

Corral-Santana, J. M., Casares, J., Munoz-Darias, T., Bauer, F. E., MartinezPais, I. G., and Russell, D. M. (2016). BlackCAT: a catalogue of stellar-mass black holes in X-ray transients. Astron. Astrophys. 587:A61. doi: 10.1051/0004-6361/201527130

Cotesta, R., Buonanno, A., Bohe, A., Taracchini, A., Hinder, I., and Ossokine, S. (2018). Enriching the symphony of gravitational waves from binary black holes by tuning higher harmonics. Phys. Rev. D 98:084028. doi: 10.1103/PhysRevD.98.084028

Eardley, D. M., Lee, D. L., and Lightman, A. P. (1973). Gravitational-wave observations as a tool for testing relativistic gravity. Phys. Rev. D 8, 3308-3321. doi: 10.1103/PhysRevD.8.3308

Eda, K., Itoh, Y., Kuroyanagi, S., and Silk, J. (2015). Gravitational waves as a probe of dark matter minispikes. Phys. Rev. D 91:044045. doi: 10.1103/PhysRevD.91.044045

Einstein, A. (1915). On the general theory of relativity. Sitzungsber. Preuss. Akad. Wiss. Berlin 1915, 778-786.

Einstein, A. (1918). On gravitational waves. Sitzungsber. Preuss. Akad. Wiss. Berlin 1918, 154-167.

Farr, W. M., Kremer, K., Lyutikov, M., and Kalogera, V. (2011). Spin tilts in the double pulsar reveal supernova spin angular-momentum production. Astrophys. J. 742:81. doi: 10.1088/0004-637X/742/2/81

Field, S. E., Galley, C. R., Hesthaven, J. S., Kaye, J., and Tiglio, M. (2014). Fast prediction and evaluation of gravitational waveforms using surrogate models. Phys. Rev. X 4:031006. doi: 10.1103/PhysRevX.4.031006

Garcia-Quiros, C., Colleoni, M., Husa, S., Estelles, H., Pratten, G., Ramos-Buades, A., et al. (2020). IMRPhenomXHM: A multi-mode frequency-domain model for the gravitational wave signal from non-precessing black-hole binaries. 
Gerosa, D., and Berti, E. (2017). Are merging black holes born from stellar collapse or previous mergers? Phys. Rev. D 95:124046. doi: 10.1103/PhysRevD.95.124046

Ghosh, A., Del Pozzo, W., and Ajith, P. (2016a). Estimating parameters of binary black holes from gravitational-wave observations of their inspiral, merger and ringdown. Phys. Rev. D 94:104070. doi: 10.1103/PhysRevD.94.104070

Ghosh, A., Ghosh, A., Johnson-Mcdaniel, N. K., Mishra, C. K., Ajith, P., Del Pozzo, W., et al. (2016b). Testing general relativity using golden black-hole binaries. Phys. Rev. D 94:021101. doi: 10.1103/PhysRevD.94.021101

Gold, R., and Brügmann, B. (2013). Eccentric black hole mergers and zoomwhirl behavior from elliptic inspirals to hyperbolic encounters. Phys. Rev. D 88:064051. doi: 10.1103/PhysRevD.88.064051

Hannam, M., Schmidt, P., Bohé, A., Haegel, L., Husa, S., Ohme, F., et al. (2014). Simple model of complete precessing black-hole-binary gravitational waveforms. Phys. Rev. Lett. 113:151101. doi: 10.1103/PhysRevLett.113.151101

Harms, E., Lukes-Gerakopoulos, G., Bernuzzi, S., and Nagar, A. (2016). Asymptotic gravitational wave fluxes from a spinning particle in circular equatorial orbits around a rotating black hole. Phys. Rev. D 93:044015. doi: 10.1103/PhysRevD.93.044015

Healy, J., Lousto, C. O., Lange, J., O'Shaughnessy, R., Zlochower, Y., and Campanelli, M. (2019). Second RIT binary black hole simulations catalog and its application to gravitational waves parameter estimation. Phys. Rev. D 100:024021. doi: 10.1103/PhysRevD.100.024021

Hinder, I., Kidder, L. E., and Pfeiffer, H. P. (2018). Eccentric binary black hole inspiral-merger-ringdown gravitational waveform model from numerical relativity and post-Newtonian theory. Phys. Rev. D 98:044015. doi: 10.1103/PhysRevD.98.044015

Hinderer, T., and Babak, S. (2017). Foundations of an effective-one-body model for coalescing binaries on eccentric orbits. Phys. Rev. D 96:104048. doi: 10.1103/PhysRevD.96.104048

Huang, Y., Middleton, H., Ng, K. K. Y., Vitale, S., and Veitch, J. (2018). Characterization of low-significance gravitational-wave compact binary sources. Phys. Rev. D 98:123021. doi: 10.1103/PhysRevD.98.123021

Huerta, E.A., Haas, R., Habib, S., Gupta, A., Rebei, A., Chavva, V., et al. (2019). Physics of eccentric binary black hole mergers: a numerical relativity perspective. Phys. Rev. D 100:064003. doi: 10.1103/PhysRevD.100.064003

Hughes, S. A., and Menou, K. (2005). Golden binaries for LISA: robust probes of strong-field gravity. Astrophys. J. 623, 689-699. doi: 10.1086/ 428826

Husa, S., Khan, S., Hannam, M., Pürrer, M., Ohme, F., Forteza, X. J., et al. (2016). Frequency-domain gravitational waves from nonprecessing black-hole binaries. I. New numerical waveforms and anatomy of the signal. Phys. Rev. D 93:044006. doi: 10.1103/PhysRevD.93.044006

Jani, K., Healy, J., Clark, J. A., London, L., Laguna, P., and Shoemaker, D. (2016). Georgia tech catalog of gravitational waveforms. Class. Quant. Grav. 33:204001. doi: 10.1088/0264-9381/33/20/204001

Kalogera, V. (2000). Spin orbit misalignment in close binaries with two compact objects. Astrophys. J. 541, 319-328. doi: 10.1086/309400

Kamaretsos, I., Hannam, M., Husa, S., and Sathyaprakash, B. S. (2012). Black-hole hair loss: learning about binary progenitors from ringdown signals. Phys. Rev. D 85:024018. doi: 10.1103/PhysRevD.85.024018

Khan, S., Chatziioannou, K., Hannam, M., and Ohme, F. (2019). Phenomenological model for the gravitational-wave signal from precessing binary black holes with two-spin effects. Phys. Rev. D 100:024059. doi: 10.1103/PhysRevD.100.024059

Khan, S., Husa, S., Hannam, M., Ohme, F., Pürrer, M., Jiménez Forteza, X., et al. (2016). Frequency-domain gravitational waves from nonprecessing black-hole binaries. II. A phenomenological model for the advanced detector era. Phys. Rev. D 93:044007. doi: 10.1103/PhysRevD.93.044007

Kidder, L. E. (1995). Coalescing binary systems of compact objects to postNewtonian 5/2 order. 5. Spin effects. Phys. Rev. D52:821-847. doi: 10.1103/PhysRevD.52.821

Klimenko, S., Vedovato, G., Drago, M., Salemi, F., Tiwari, V., Prodi, G. A., et al. (2016). Method for detection and reconstruction of gravitational wave transients with networks of advanced detectors. Phys. Rev. D 93:042004. doi: 10.1103/PhysRevD.93.042004

Kokkotas, K. D., and Schmidt, B. G. (1999). Quasinormal modes of stars and black holes. Living Rev. Relat. 2:2. doi: 10.12942/lrr-1999-2
Lewis, A. G. M., Zimmerman, A., and Pfeiffer, H. P. (2017). Fundamental frequencies and resonances from eccentric and precessing binary black hole inspirals. Class. Quant. Grav. 34:124001. doi: 10.1088/1361-6382/aa66f4

LIGO Scientific Collaboration, Virgo Collaboration (2018). GWTC-1. doi: $10.7935 / 82 \mathrm{H} 3-\mathrm{HH} 23$

LIGO Scientific, Virgo Collaboration. GraceDB. Available online at: https:// gracedb.ligo.org/superevents/public/O3/

Loffler, F., Faber, J., Bentivegna, E., Bode, T., Diener, P., Haas, R., et al. (2012). The Einstein Toolkit: a community computational infrastructure for relativistic astrophysics. Class. Quant. Grav. 29:115001. doi: $10.1088 / 0264-9381 / 29 / 11 / 115001$

London, L., Shoemaker, D., and Healy, J. (2014). Modeling ringdown: beyond the fundamental quasinormal modes. Phys. Rev. D 90(12):124032. doi: $10.1103 /$ PhysRevD.90.124032

Mandel, I., and O'Shaughnessy, R. (2010). Compact binary coalescences in the band of ground-based gravitational-wave detectors. Classic. Quant. Grav. 27:114007. doi: 10.1088/0264-9381/27/11/114007

Marchant, P., Renzo, M., Farmer, R., Pappas, K. M. W., Taam, R. E., de Mink, S., et al. (2018). Pulsational pair-instability supernovae in very close binaries. Astrophys. J. 882:36. doi: 10.3847/1538-4357/ab3426

Messick, C., Blackburn, K., Brady, Brockill, P., Cannon, K., Cariou, R., et al. (2017). Analysis framework for the prompt discovery of compact binary mergers in gravitational-wave data. Phys. Rev. D 95:042001. doi: 10.1103/PhysRevD.95.042001

Mirshekari, S., Yunes, N., and Will, C. M. (2012). Constraining generic lorentz violation and the speed of the graviton with gravitational waves. Phys. Rev. D 85:024041. doi: 10.1103/PhysRevD.85.024041

Moore, B., and Yunes, N. (2020). Constraining gravity with eccentric gravitational waves: projected upper bounds and model selection. arXiv [Preprint]. doi: 10.1088/1361-6382/ab8bb6

Mroue, A. H., Scheel, M. A., Szilagyi, B., Pfeiffer, H. P., Boyle, M., Hemberger, D. A., et al. (2013). Catalog of 174 binary black hole simulations for gravitational wave astronomy. Phys. Rev. Lett. 111:241104. doi: 10.1103/PhysRevLett.111.241104

Nagar, A., Bernuzzi, S., Del Pozzo, W., Riemenschneider, G., Akcay, S., Carullo, G., et al. (2018). Time-domain effective-one-body gravitational waveforms for coalescing compact binaries with nonprecessing spins, tides and self-spin effects. Phys. Rev. D 98:104052. doi: 10.1103/PhysRevD.98.104052

Nagar, A., Pratten, G., Riemenschneider, G., and Gamba, R. (2020a). Multipolar effective one body model for nonspinning black hole binaries. Phys. Rev. D 101:024041. doi: 10.1103/PhysRevD.101.024041

Nagar, A., Riemenschneider, G., Pratten, G., Rettegno, P., and Messina, F. (2020b). A multipolar effective one body waveform model for spin-aligned black hole binaries. arXiv [Preprint]. arXiv:2001.09082.

Nitz, A. H., Dent, T., Davies, G. S., Kumar, S., Capano, C. D., Harry, I., et al. (2019a). 2-OGC: open gravitational-wave catalog of binary mergers from analysis of public Advanced LIGO and Virgo data. arXiv [Preprint]. arXiv:1910.05331. doi: 10.3847/1538-4357/ab733f

Nitz, A. H., Dent, T., Davies, G. S., Kumar, S., Capano, C. D., Harry, I., et al. (2019b). Posterior Samples. Available online at: https://github.com/gwastro/2ogcl

Nitz, A. H., Harry, I. W., Willis, J. L., Biwer, C. M., Brown, D. A., Pekowsky, L. P., et al. (2017). PyCBC Software. GitHub Repository. doi: 10.5281/zenodo.344823

Okounkova, M., Stein, L. C., Moxon, J., Scheel, M. A., and Teukolsky, S. A. (2019). Numerical relativity simulation of GW150914 beyond general relativity. arXiv [Preprint]. arXiv:1911.02588. doi: 10.1103/PhysRevD.101. 104016

O'Shaughnessy, R., Vaishnav, B., Healy, J., Meeks, Z., and Shoemaker, D. (2011). Efficient asymptotic frame selection for binary black hole spacetimes using asymptotic radiation. Phys. Rev. D 84:124002. doi: 10.1103/PhysRevD.84.124002

Ossokine, S., Buonanno, A., Marsat, S., Cotesta, R., Babak, S., Dietrich, T., et al. (2020). Multipolar effective-one-body waveforms for precessing binary black holes: construction and validation. arXiv [Preprint]. arXiv:2004.09442.

Özel, F., Psaltis, D., Narayan, R., and McClintock, J. E. (2010). The black hole mass distribution in the galaxy. Astrophys. J. 725, 1918-1927. doi: $10.1088 / 0004-637 \mathrm{X} / 725 / 2 / 1918$

Pan, Y., Buonanno, A., Taracchini, A., Kidder, L. E., Mroué, A. H., Pfeiffer, H. P., et al. (2014). Inspiral-merger-ringdown waveforms of spinning, 
precessing black-hole binaries in the effective-one-body formalism. Phys. Rev. D 89:084006. doi: 10.1103/PhysRevD.89.084006

Poisson, E., Pound, A., and Vega, I. (2011). The Motion of point particles in curved spacetime. Living Rev. Relat. 14:7. doi: 10.12942/lrr-2011-7

Pound, A., Wardell, B., Warburton, N., and Miller, J. (2020). Second-order selfforce calculation of the gravitational binding energy in compact binaries. Phys. Rev. Lett. 124:021101. doi: 10.1103/PhysRevLett.124.021101

Pratten, G., García-Quirós, C., Colleoni, M., Ramos-Buades, A., Estellés, H., Mateu-Lucena, M., et al. (2020a). Let's twist again: computationally efficient models for the dominant and sub-dominant harmonic modes of precessing binary black holes. arXiv [Preprint]. arXiv:2004.06503.

Pratten, G., Husa, S., Garcia-Quiros, C., Colleoni, M., Ramos-Buades, A., Estelles, H., et al. (2020b). Setting the cornerstone for the IMRPhenomX family of models for gravitational waves from compact binaries: the dominant harmonic for non-precessing quasi-circular black holes. arXiv [preprint]. arXiv:2001.11412. Available online at: https://dcc.ligo.org/LIGO-P2000018

Pretorius, F. (2005). Evolution of binary black hole spacetimes. Phys. Rev. Lett. 95:121101. doi: 10.1103/PhysRevLett.95.121101

Punturo, M., Abernathy, M., Acernese, F., Allen, B., Andersson, N., Arun, K., et al. (2010). The Einstein telescope: a third-generation gravitational wave observatory. Classic. Quant. Grav. 27:194002. doi: 10.1088/0264-9381/27/19/194002

Racine, É. (2008). Analysis of spin precession in binary black hole systems including quadrupole-monopole interaction. Phys. Rev. D 78:044021. doi: 10.1103/PhysRevD.78.044021

Ramos-Buades, A., Husa, S., Pratten, G., Estellés, H., García-Quirós, C., MateuLucena, M., et al. (2020). First survey of spinning eccentric black hole mergers: Numerical relativity simulations, hybrid waveforms, and parameter estimation. Phys. Rev. D 101:083015. doi: 10.1103/PhysRevD.101.083015

Reitze, D., Adhikari, R. X., Ballmer, S., Barish, B., Barsotti, L., Billingsley,G., et al. (2019). Cosmic Explorer: the U.S. contribution to gravitational-wave astronomy beyond LIGO. Bull. Am. Astron. Soc. 51:035.

Rodriguez, C. L., Zevin, M., Pankow, C., Kalogera, V., and Rasio, F. A. (2016). Illuminating black hole binary formation channels with spins in advanced LIGO. Astrophys. J. Lett. 83:L2. doi: 10.3847/2041-8205/832/1/L2

Santamaría, L., Ohme, F., Ajith, P., Brugmann, B., Dorband, N., Hannam, M., et al. (2010). Matching post-Newtonian and numerical relativity waveforms: systematic errors and a new phenomenological model for non-precessing black hole binaries. Phys. Rev. D 82:064016. doi: 10.1103/PhysRevD.82.064016

Sathyaprakash, B., Abernathy, M., Acernese, F., Ajith, P., Allen, B., Amaro-Seoane, P., et al. (2012). Scientific objectives of Einstein telescope. Classic. Quant. Grav. 29:124013. doi: 10.1088/0264-9381/30/7/079501

Sathyaprakash, B. S., and Dhurandhar, S. V. (1991). Choice of filters for the detection of gravitational waves from coalescing binaries. Phys. Rev. D 44, 3819-3834. doi: 10.1103/PhysRevD.44.3819

Scheel, M. A., Pfeiffer, H. P., Lindblom, L., Kidder, L. E., Rinne, O., and Teukolsky, S. A. (2006). Solving Einstein's equations with dual coordinate frames. Phys. Rev. D 74:104006. doi: 10.1103/PhysRevD.74.104006

Schmidt, P., Hannam, M., and Husa, S. (2012). Towards models of gravitational waveforms from generic binaries: a simple approximate mapping between precessing and non-precessing inspiral signals. Phys. Rev. D 86:104063. doi: 10.1103/PhysRevD.86.104063

Schmidt, P., Hannam, M., Husa, S., and Ajith, P. (2011). Tracking the precession of compact binaries from their gravitationalwave signal. Phys. Rev. D 84:024046. doi: 10.1103/PhysRevD.84. 024046

Sennett, N., Marsat, S., and Buonanno, A. (2016). Gravitational waveforms in scalar-tensor gravity at 2PN relative order. Phys. Rev. D 94:084003. doi: 10.1103/PhysRevD.94.084003

Sesana, A. (2016). Prospects for multiband gravitational-wave astronomy after GW150914. Phys. Rev. Lett. 116:231102. doi: 10.1103/PhysRevLett.116.231102

Sperhake, U. (2007). Binary black-hole evolutions of excision and puncture data. Phys. Rev. D 76:104015. doi: 10.1103/PhysRevD.76. 104015

Sperhake, U., Berti, E., Cardoso, V., González, J. A., Brügmann, B., and Ansorg, M. (2008). Eccentric binary black-hole mergers: the transition from inspiral to plunge in general relativity. Phys. Rev. D 78:064069. doi: 10.1103/PhysRevD.78.064069
Stevenson, S., Berry, C. P., and Mandel, I. (2017). Hierarchical analysis of gravitational-wave measurements of binary black hole spinorbit misalignments. Mon. Not. R. Astron. Soc. 471, 2801-2811. doi: $10.1093 / \mathrm{mnras} / \mathrm{stx} 1764$

Szilágyi, B., Blackman, J., Buonanno, A., Taracchini, A., Pfeiffer, H. P., Scheel, M. A., et al. (2015). Approaching the post-newtonian regime with numerical relativity: a compact-object binary simulation spanning 350 gravitational-wave cycles. Phys. Rev. Lett. 115:031102. doi: 10.1103/PhysRevLett.115.031102

Teukolsky, S. A. (1973). Perturbations of a rotating black hole. 1. Fundamental equations for gravitational electromagnetic and neutrino field perturbations. Astrophys. J. 185, 635-647. doi: 10.1086/152444

Usman, S. A., Nitz, A. H., Harry, I. W., Biwer, C. W., Brown, D. A., Cabero, M., et al. (2016). The PyCBC search for gravitational waves from compact binary coalescence. Classic. Quant. Grav. 33:215004. doi: 10.1088/0264-9381/33/21/215004

Vaishnav, B., Hinder, I., Herrmann, F., and Shoemaker, D. (2007). Matched filtering of numerical relativity templates of spinning binary black holes. Phys. Rev. D 76:084020. doi: 10.1103/PhysRevD.76.084020

Varma, V., Field, S. E., Scheel, M. A., Blackman, J., Gerosa, D., Stein, L. C., et al. (2019a). Surrogate models for precessing binary black hole simulations with unequal masses. Phys. Rev. Res. 1:033015. doi: 10.1103/PhysRevResearch.1.033015

Varma, V., Field, S. E., Scheel, M. A., Blackman, J., Kidder, L. E., and Pfeiffer, H. P. (2019b). Surrogate model of hybridized numerical relativity binary black hole waveforms. Phys. Rev. D 99:064045. doi: 10.1103/PhysRevD.99.064045

Veitch, J., Raymond, V., Blackburn, K., and Smith, R. (2015). Parameter estimation for compact binaries with ground-based gravitational-wave observations using the LALInference software library. Phys. Rev. D 91:042003. doi: 10.1103/PhysRevD.91.042003

Venumadhav, T., Zackay, B., Roulet, J., Dai, L., and Zaldarriaga, M. (2019a). New binary black hole mergers in the second observing run of advanced LIGO and advanced Virgo. Phys. Rev. D 101:083030. doi: 10.1103/PhysRevD.101.083030

Venumadhav, T., Zackay, B., Roulet, J., Dai, L., and Zaldarriaga, M. (2019b). New search pipeline for compact binary mergers: Results for binary black holes in the first observing run of Advanced LIGO. Phys. Rev. D 100:023011. doi: 10.1103/PhysRevD.100.023011

Venumadhav, T., Zackay, B., Roulet, J., Dai, L., and Zaldarriaga, M. (2019c). O2 Samples. Available online at: https://github.com/jroulet/O2_samples

Verde, L., Treu, T., and Riess, A. G. (2019). Tensions between the early and the late universe. arXiv [Preprint]. doi: 10.1038/s41550-019-0902-0

Witek, H., Gualtieri, L., Pani, P., and Sotiriou, T. P. (2019). Black holes and binary mergers in scalar Gauss-Bonnet gravity: scalar field dynamics. Phys. Rev. D 99:064035. doi: 10.1103/PhysRevD.99.064035

Woosley, S. E. (2016). The progenitor of Gw150914. Astrophys. J. Lett. 824:L10. doi: $10.3847 / 2041-8205 / 824 / 1 / \mathrm{L} 10$

Yunes, N., and Pretorius, F. (2009). Fundamental theoretical bias in gravitational wave astrophysics and the parameterized post-Einsteinian framework. Phys. Rev. D 80:122003. doi: 10.1103/PhysRevD.80.122003

Yunes, N., Yagi, K., and Pretorius, F. (2016). Theoretical physics implications of the binary black-hole mergers GW150914 and GW151226. Phys. Rev. D 94:084002. doi: 10.1103/PhysRevD.94.084002

Zackay, B., Dai, L., and Venumadhav, T. (2018). Relative binning and fast likelihood evaluation for gravitational wave parameter estimation. arXiv [Preprint]. arXiv:1806.08792.

Zackay, B., Venumadhav, T., Dai, L., Roulet, J., and Zaldarriaga, M. (2019). Highly spinning and aligned binary black hole merger in the Advanced LIGO first observing run. Phys. Rev. D 100:023007. doi: 10.1103/PhysRevD.100.023007

Conflict of Interest: The author declares that the research was conducted in the absence of any commercial or financial relationships that could be construed as a potential conflict of interest.

Copyright (C) 2020 Schmidt. This is an open-access article distributed under the terms of the Creative Commons Attribution License (CC BY). The use, distribution or reproduction in other forums is permitted, provided the original author(s) and the copyright owner(s) are credited and that the original publication in this journal is cited, in accordance with accepted academic practice. No use, distribution or reproduction is permitted which does not comply with these terms. 